\title{
Collaborative Optimization of Stop Schedule Plan and Ticket Allotment for the Intercity Train
}

\author{
Xichun Chen ${ }^{1}$ and Junli Wang ${ }^{2}$ \\ ${ }^{1}$ School of Traffic and Transportation, Lanzhou Jiaotong University, Lanzhou 730070, China \\ ${ }^{2}$ Gansu Province Product Quality Supervision and Inspection Center, Lanzhou 730070, China \\ Correspondence should be addressed to Xichun Chen; chenxichun@163.com
}

Received 13 July 2015; Revised 14 November 2015; Accepted 16 November 2015

Academic Editor: Filippo Cacace

Copyright (c) $2016 \mathrm{X}$. Chen and J. Wang. This is an open access article distributed under the Creative Commons Attribution License, which permits unrestricted use, distribution, and reproduction in any medium, provided the original work is properly cited.

\begin{abstract}
As regards the ticket allotment issue of the intercity passenger corridor designed for different train grades, the matching relationship between the ticket allotment and the passenger flow demand is studied. The passenger flow conversion equation which is based on the collaborative optimization of the intercity train stop schedule plan and ticket allotment is established. Then the mathematical model aiming at the maximum revenue of intercity train system and the highest satisfaction from the passengers is established. The particle swarm harmony search algorithm is designed to solve the model. The example verifies the effectiveness of the model and algorithm, which indicates that, through the collaborative optimization of the stop schedule plan and ticket allotment for different grades intercity trains, the sectional utilization rate of the train can be improved; meanwhile, the optimum matching between the intercity train revenue and the passenger satisfaction can be realized.
\end{abstract}

\section{Introduction}

Intercity train ticket allotment is the important content of railway revenue management, which, with the basis of the passenger travelling demand at all stations along the corridor and the comprehensive consideration on the influence of the operation plan of different grades intercity trains, aims at the optimum matching between sectional tickets and the passenger demand.

Revenue management [1-3] was firstly applied to air transport industry in America in the 1970s, with the purpose of selling the passenger seats to the appropriate customer at the appropriate time with appropriate price by using the pricing strategy as the core, which helped to match the air transport capacity with the potential passenger demand in the market segmentation and thus realized the maximum revenue. One of its core issues is seat inventory control [4]. In recent years, some scholars have studied the railway revenue management by referring to the aviation management mode and introduced the concept of seat inventory control to the railway passenger transport. Ciancimino et al. [5] and You [6] studied the seat inventory control issue of the single grade train and 2-grade trains. Bao [7] studied seat inventory control issue of the multiple grades trains. All of the previous researches are good solutions to sectional seat inventory control with the same origin-destination. Compared with pointto-point passenger transportation mode like air transport, the significant difference of railway transport lies in that there are many stations along the railway, which demands to take sectional seat inventory control, the influence of stop schedule plan on passenger flow, and seat inventory control of multiple grades trains in different sections into consideration. Huang and Peng [8] and Peng and Zhu [9] studied the influence of different stop schedule plans on passenger flow and put forward passenger conversion equation. But its model implied hypothesis in which the amount of tickets can fully meet all passenger demands without taking induction effect caused by different ticket allotment for passenger flow. Therefore, facing intercity passenger rail corridor with coexisting high-speed trains and ordinary ones, the dynamic relationship between passenger flow demands, stop schedule plan, and ticket allotment should be discussed to realize the balanced distribution of the amount of tickets in different grades trains and different sections. 


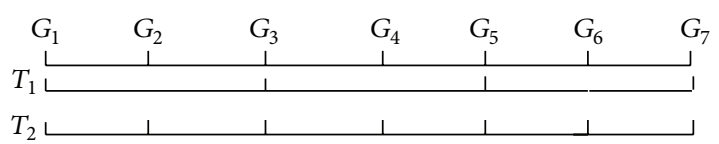

FIGURE 1: Stop schedule plan of the intercity passenger rail corridor.

In the traditional seat inventory control, the basic ticket plan in the train diagram phase [10] and ticket preallotment plan in the presale period [11] are studied by stages through the ticket-selling strategies such as setting up the stations of restricted tickets sale, reusing tickets in short distance, sharing tickets, and reusing seats [12]. The features of passenger flow regularity of the intercity railway and public transport type decide the possibility of combining the basic ticket plan and ticket preallotment plan together. Through collaborative optimization of the two plans, the equilibrium distribution of tickets on corresponding sections can be realized at a time.

The main mode of intercity railway is ordinary train and high-speed trains in parallel; based on this, the paper investigates the influence of the stop schedule plan and ticket allotment on the passenger flow demand from the perspective of the passenger behavior choice, establishes the passenger flow conversion equation to depict the dynamic relationship, and sets up a multiobjective model to collaboratively optimize the stop schedule plan and ticket allotment strategy so as to realize the optimum matching between the passenger flow demand and the system revenue.

\section{Process of Passenger Travelling Behavior Choice}

Build an intercity passenger rail corridor as it is shown in Figure 1 to analyze the passenger travelling choice process.

Suppose there are trains of two grades in the intercity passenger rail corridor. Take $G_{1}$ station for the starting station and $G_{7}$ station for the terminal station. Let $T_{1}$ denote highspeed passenger train, of which stop stations are $G_{3}$ and $G_{5}$, facing a high-speed passenger flow; let $T_{2}$ denote ordinary passenger train, of which stop schedule plan of stopping at every station from $G_{1}$ to $G_{7}$ is adopted, facing an ordinaryspeed passenger flow.

In view of the two different stop schedule plans above, at first the passenger should consider if there is the direct train and ticket corresponding to its passenger flow type at the destination; if so, the passenger can make the choice accordingly. Take the high-speed passenger flow as an example to analyze the passenger behavior choice process when there is no direct train or no ticket corresponding to its own passenger flow type. As is shown in Figure 1, high-speed passengers from $G_{1}$ to $G_{4}$ cannot choose $T_{1}$ to arrive at the destination directly. This part of the passenger flow will be converted. They may choose $T_{2}$ to arrive directly. They may also choose $T_{1}$ to station $G_{3}$ and then transfer to $T_{2}$ to arrive at $G_{4}$. Whether or not these passengers will choose $T_{2}$ depends on whether or not there are tickets from $G_{1}$ to $G_{4}$. Likewise, whether or not the passengers will choose to transfer at $G_{3}$ depends on whether or not there are tickets from $G_{1}$ to $G_{3}$ by $T_{1}$ and from $G_{3}$ to $G_{4}$ by $T_{2}$.
From the above analysis, the passenger's choice of direct train depends on its own passenger flow type and sectional ticket allotment and the passenger's choice of transfer scheme depends on the train ticket allotment at the transfer station. In the process of the passenger behavior choice, different stop schedule plans and ticket allotments will cause the ceaseless conversion of initial passenger flow and the dynamism of passenger flow which, in turn, will affect the stop schedule plan and ticket allotment. In this way, the linkage and collaboration between passenger flow demand and ticket allotment can be realized.

\section{Model}

\subsection{Research Hypothesis}

(1) Hypothesis on the Closure of Railway Passenger Transport Corridor System. The change of system state only depends on the change of the internal factors, instead of being influenced by the external factors.

(2) Transfer Hypothesis. Passengers give priority to the passenger train which is corresponding to their passenger flow type. If the direct arrival cannot be adopted, then the passengers will change the train grade or transfer. If there are two or more transfer stations, the passengers will choose the transfer station which is closest to the destination for one transfer in this paper.

3.2. Parameter Definition. Assume that the station set of intercity passenger rail corridor is $A=\{1,2, \ldots, n\}$, in which $i, j$ is marked as the station, $i, j \in A$; assume that the train set on the line is $T=\left\{T_{1}, T_{2}\right\}$, in which $T_{1}$ denotes high-speed passenger train and $T_{2}$ denotes ordinary-speed passenger train. Train $k \in T$, then $t_{i}^{k}$ denotes stopping time of Train $k$ in the $i$ station, $t_{i j}^{k}$ denotes travelling time of Train $k$ in the $i, j$ section, $P_{i j}^{k}$ denotes ticket price of Train $k$ in the $i, j$ section, and $B^{k}$ denotes seating capacity of Train $k$. Set the $0-1$ variable $\alpha_{i}^{k}$ indicating whether Train $k$ stops at $i$ station. When $\alpha_{i}^{k}=1$, Train $k$ stops at $i$ station; otherwise, it does not. If there is station $m$ between $i$ and $j$, when $\alpha_{m}^{T_{1}}=\alpha_{m}^{T_{2}}=1$, the station can be used as transfer station for $T_{1}$ and $T_{2}$, and variable $\theta_{i j}^{k}$ indicates ticket number of Train $k$ between $i$ and $j$.

3.3. Decision Condition. Passengers give priority to the passenger train which is corresponding to their passenger flow type. If the direct arrival cannot be achieved, then the passengers will change the train grade or transfer. When there are multiple transfer stations, passengers will choose the transfer station which can maximize their travel utilities according to their passenger flow type. Passenger travel utility includes the discounted cost as per the passenger travel time and the train ticket cost. The calculation formula is shown as in

$$
\begin{aligned}
U_{m}= & E^{T_{1}}\left(t_{m}^{\prime}+t_{i m}^{T_{1}}+t_{m j}^{T_{2}}+\sum_{i=2}^{m-1} \alpha_{i}^{T_{1}} t_{i}^{T_{1}}+\sum_{i=m}^{j-1} \alpha_{i}^{T_{2}} t_{i}^{T_{2}}\right) \\
& +P_{i m}^{T_{1}}+P_{m j}^{T_{2}} .
\end{aligned}
$$


In formula, $U_{m}$ indicates the travel utility when passengers choose station $m$ to transfer; $t_{m}^{\prime}$ indicates the transfer time when passengers transfer in station $m$, which is decided by departure time interval of two-grade trains in the station. To simplify the model, this paper takes it as a fixed value. $E^{T_{1}}$ indicates the high-speed passenger travel time value.

When plans of transfer and changing the train grade are both feasible, passengers will make a comparison according to the travel utility computational formula. When the transfer utility is greater than the utility of changing the train grade in the transfer station $m$, that is, formula (2) is satisfied, the high-speed passengers will choose to transfer at the transfer station $m$, otherwise, change the train grade:

$$
U_{m}>E^{T_{1}}\left(t_{i j}^{T_{2}}+\sum_{i=2}^{j-1} \alpha_{i}^{T_{2}} t_{i}^{T_{2}}\right)+P_{i j}^{T_{2}} .
$$

Namely, in the face of choosing multiple transfer stations and changing the train grade, travelers will always choose the trip mode which can maximize their travel utilities.

\subsection{Passenger Flow Conversion Equation. For passengers} between $i$ and $j, D=\left(d_{i j}^{k}\right)_{n \times n}$ indicates the passenger flow OD matrix and $d_{i j}^{k}$ indicates the initial passenger flow from Train $k$. Since the influence of the stop schedule plan and ticket allotment restriction on the initial passenger flow will lead to the passenger flow conversion, $h_{i j}^{k}$ is used to indicate the actual passenger flow converted.

When $d_{i j}^{T_{1}}>\theta_{i j}^{T_{1}}, d_{i j}^{T_{2}}>\theta_{i j}^{T_{2}}$, the passenger transport capacity can only be ensured by running extra trains.

When $d_{i j}^{T_{1}} \leq \theta_{i j}^{T_{1}}, d_{i j}^{T_{2}} \leq \theta_{i j}^{T_{2}}$, passengers will choose trains according to their passenger flow types.

When $d_{i j}^{T_{1}}>\theta_{i j}^{T_{1}}, d_{i j}^{T_{2}}<\theta_{i j}^{T_{2}}$, the process of passenger flow conversion is analyzed as follows.

When there is no transfer station $m$ between $i$ and $j$ or the transfer station has no tickets, the remaining highspeed passengers can only choose to take $T_{2}$. At this moment, the actual number of high-speed passengers is the actual number of high-speed tickets allotted between $i$ and $j$, while the actual number of passengers for ordinary-speed train is the smaller value between the sum of the ordinary-speed passengers converted from the high-speed passengers and the current ordinary-speed passengers, and the number of ordinary-speed tickets allotted between $i$ and $j$. Namely,

$$
\begin{aligned}
& h_{i j}^{T_{1}}=\theta_{i j}^{T_{1}}, \\
& h_{i j}^{T_{2}}=\min \left[d_{i j}^{T_{2}}+\left(d_{i j}^{T_{1}}-\theta_{i j}^{T_{1}}\right), \theta_{i j}^{T_{2}}\right] .
\end{aligned}
$$

When there is a transfer station $m$ between $i$ and $j$ and the station has tickets, the passenger flow will transfer at the transfer station, as is shown in Figure 2.

Now, the remaining high-speed passengers between $i$ and $j$ are $d_{i j}^{T_{1}}-\theta_{i j}^{T_{1}}$, the remaining high-speed tickets between $i$ and $m$ are $\theta_{i m}^{T_{1}}-d_{i m}^{T_{1}}$, and the remaining ordinary-speed tickets between $m$ and $j$ are $\theta_{m j}^{T_{2}}-d_{m j}^{T_{2}}$. Compare the numerical

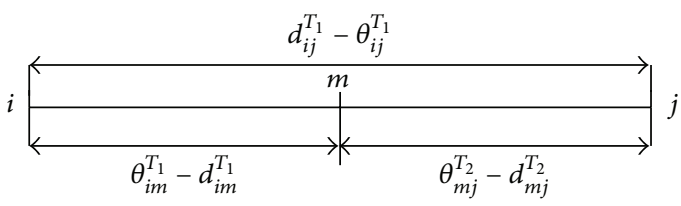

FIGURE 2: Relational graph of remaining passengers and remaining tickets at the transfer station.

relationship among them and then the actual number of passengers at each section can be acquired after conversion.

$$
\begin{aligned}
\text { When } \min \left(\theta_{i m}^{T_{1}}-d_{i m}^{T_{1}}, \theta_{m j}^{T_{2}}-d_{m j}^{T_{2}}, d_{i j}^{T_{1}}-\theta_{i j}^{T_{1}}\right)=d_{i j}^{T_{1}}-\theta_{i j}^{T_{1}}, \\
h_{i m}^{T_{1}}=d_{i m}^{T_{1}}+d_{i j}^{T_{1}}-\theta_{i j}^{T_{1}} \\
h_{m j}^{T_{1}}=\theta_{m j}^{T_{1}} \\
h_{i m}^{T_{2}}=d_{i m}^{T_{2}} \\
h_{m j}^{T_{2}}=d_{m j}^{T_{2}}+d_{i j}^{T_{1}}-\theta_{i j}^{T_{1}}
\end{aligned}
$$

When $\min \left(\theta_{i m}^{T_{1}}-d_{i m}^{T_{1}}, \theta_{m j}^{T_{2}}-d_{m j}^{T_{2}}, d_{i j}^{T_{1}}-\theta_{i j}^{T_{1}}\right)=\theta_{i m}^{T_{1}}-d_{i m}^{T_{1}}$

$$
\begin{aligned}
& h_{i m}^{T_{1}}=\theta_{i m}^{T_{1}}, \\
& h_{m j}^{T_{1}}=\theta_{m j}^{T_{1}}, \\
& h_{i m}^{T_{2}}=d_{i m}^{T_{2}}, \\
& h_{m j}^{T_{2}}=d_{m j}^{T_{2}}+\theta_{i m}^{T_{1}}-d_{i m}^{T_{1}} .
\end{aligned}
$$

$$
\begin{aligned}
\text { When } \min \left(\theta_{i m}^{T_{1}}-d_{i m}^{T_{1}}, \theta_{m j}^{T_{2}}-d_{m j}^{T_{2}}, d_{i j}^{T_{1}}-\theta_{i j}^{T_{1}}\right)=\theta_{m j}^{T_{2}}-d_{m j}^{T_{2}}, \\
h_{i m}^{T_{1}}=d_{i m}^{T_{1}}+\theta_{m j}^{T_{2}}-d_{m j}^{T_{2}}, \\
h_{m j}^{T_{1}}=\theta_{m j}^{T_{1}}, \\
h_{m j}^{T_{2}}=\theta_{m j}^{T_{2}}, \\
h_{i m}^{T_{2}}=d_{i m}^{T_{2}} .
\end{aligned}
$$

When $d_{i j}^{T_{1}}<\theta_{i j}^{T_{1}}, d_{i j}^{T_{2}}>\theta_{i j}^{T_{2}}$, the process of passenger flow conversion is analyzed as follows.

When there is no transfer station $m$ between $i$ and $j$ or the transfer station has no tickets,

$$
\begin{aligned}
& h_{i j}^{T_{2}}=\theta_{i j}^{T_{2}}, \\
& h_{i j}^{T_{1}}=\min \left[d_{i j}^{T_{1}}+\left(d_{i j}^{T_{2}}-\theta_{i j}^{T_{2}}\right), \theta_{i j}^{T_{1}}\right] .
\end{aligned}
$$

When there is a transfer station $m$ between $i$ and $j$ and the station has tickets, one has the following. 
When $\min \left(\theta_{i m}^{T_{2}}-d_{i m}^{T_{2}}, \theta_{m j}^{T_{1}}-d_{m j}^{T_{1}}, d_{i j}^{T_{2}}-\theta_{i j}^{T_{2}}\right)=d_{i j}^{T_{2}}-\theta_{i j}^{T_{2}}$,

$$
\begin{aligned}
& h_{i m}^{T_{2}}=d_{i m}^{T_{2}}+d_{i j}^{T_{2}}-\theta_{i j}^{T_{2}}, \\
& h_{m j}^{T_{2}}=\theta_{m j}^{T_{2}}, \\
& h_{i m}^{T_{1}}=d_{i m}^{T_{1}}, \\
& h_{m j}^{T_{1}}=d_{m j}^{T_{1}}+d_{i j}^{T_{2}}-\theta_{i j}^{T_{2}} .
\end{aligned}
$$

When $\min \left(\theta_{i m}^{T_{2}}-d_{i m}^{T_{2}}, \theta_{m j}^{T_{1}}-d_{m j}^{T_{1}}, d_{i j}^{T_{2}}-\theta_{i j}^{T_{2}}\right)=\theta_{i m}^{T_{2}}-d_{i m}^{T_{2}}$,

$$
\begin{aligned}
& h_{i m}^{T_{2}}=\theta_{i m}^{T_{2}}, \\
& h_{m j}^{T_{2}}=\theta_{m j}^{T_{2}}, \\
& h_{i m}^{T_{1}}=d_{i m}^{T_{1}}, \\
& h_{m j}^{T_{1}}=d_{m j}^{T_{1}}+\theta_{i m}^{T_{2}}-d_{i m}^{T_{2}} .
\end{aligned}
$$

When $\min \left(\theta_{i m}^{T_{2}}-d_{i m}^{T_{2}}, \theta_{m j}^{T_{1}}-d_{m j}^{T_{1}}, d_{i j}^{T_{2}}-\theta_{i j}^{T_{2}}\right)=\theta_{m j}^{T_{1}}-d_{m j}^{T_{1}}$,

$$
\begin{aligned}
& h_{m j}^{T_{1}}=\theta_{m j}^{T_{1}}, \\
& h_{i m}^{T_{1}}=d_{i m}^{T_{1}}, \\
& h_{i m}^{T_{2}}=d_{i m}^{T_{2}}+\theta_{m j}^{T_{1}}-d_{m j}^{T_{1}}, \\
& h_{m j}^{T_{2}}=\theta_{m j}^{T_{2}} .
\end{aligned}
$$

3.5. Objective Function. Since intercity trains operating costs involve many aspects of vehicle operation and organization management, it is rather difficult to measure accurately; hence, this paper resolves the maximum revenue of intercity passenger rail corridor system into the maximum total passenger fares and minimum total train number, objectively describing the passengers satisfaction on travel with the minimum conversion of passenger flow demand.

3.5.1. Maximum Passenger Fares of Intercity Trains. Total passenger fares of Train $k$ are

$$
M^{k}=\sum_{i=1}^{n} \sum_{j=i}^{n} P_{i j}^{k} h_{i j}^{k}
$$

Maximum passenger fares of intercity trains are

$$
\max M=\sum_{k=1}^{2} \sum_{i=1}^{n} \sum_{j=i}^{n} P_{i j}^{k} h_{i j}^{k}
$$

$M$ indicates total passenger fares of intercity trains.

3.5.2. Minimum Number of Train $k$. The maximum section of passenger flow will be used to solve the number of Train $k$ :

$$
\begin{aligned}
& \min \gamma^{k} \\
& =\left\lceil\frac{\max _{1<m<n}\left\{\sum_{i=1}^{m} \sum_{j=m+1}^{n} \alpha_{i}^{k} \cdot \alpha_{m}^{k} \cdot \alpha_{j}^{k} \cdot h_{i j}^{k}\right\}}{B^{k}}\right\rceil .
\end{aligned}
$$

$\gamma^{k}$ indicates the number of Train $k$.
3.5.3. The Highest Passenger Travel Satisfaction. Stop and transfer of trains will affect the initial passenger flow. In order to quantitatively measure the passenger flow matrix change caused by the stop schedule plan, the Generalized Euclidean distance $R_{\mathrm{OD}}$ is used to calculate the deviation between actual passenger flow matrix and initial passenger flow matrix. As for the aspect of satisfying the passengers demand conversion of passenger flow will reduce the passenger travel satisfaction, due to which the minimum Euclidean distance is set to indicate the minimum conversion of initial passenger flow to maximize passenger satisfaction:

$$
\min R_{\mathrm{OD}}=\sum_{k \in T} \sqrt{\sum_{i=1}^{n} \sum_{j=i}^{n}\left(h_{i j}^{k}-d_{i j}^{k}\right)^{2}} .
$$

\subsection{Constraint Condition}

3.6.1. Constraint of Stop Coverage. To ensure the passengers can get on and off at all the stations, constraint will be set up to ensure at least one grade train to stop in each station:

$$
\sum_{k=1}^{2} \alpha_{i}^{k} \geq 1
$$

3.6.2. Constraint of Tickets. Sectional ticket allotment will not exceed the train seating capacity:

$$
0 \leq \frac{\sum_{i=1}^{n} \sum_{j=i}^{n} \theta_{i j}^{k}}{\gamma^{k}} \leq B^{k} .
$$

\section{Solution Algorithm}

This model is a complicated multiobjective model with the general objective function taken as $\max F=\left[\sum_{k=1}^{2}\left(M^{k} / \gamma^{k}\right)\right] /$ $R_{\mathrm{OD}}$. Setting adjustment weighting factors for system revenue $\sum_{k=1}^{2}\left(M^{k} / \gamma^{k}\right)$ and passenger demand conversion $R_{\mathrm{OD}}$ can influence the result of stop schedule plan and ticket allotment. All the weighting factors take 1 in this paper.

In order to improve the algorithm applicability, the particle swarm harmony search algorithm (15)-(16) (PSOHS) is used for the solution. The harmony search algorithm has the advantage which is better than the local optimum and the particle swarm algorithm has the characteristics of fast speed and directivity, based on which the search efficiency can be improved. Algorithm process is as follows.

Step 1 (basic parameters of PSO-HS algorithm are determined). Harmony memory size is HMS; number of variables is $N$; variable solution space is $W$; harmony memory retention probability is HMCR; value range of tone control probability PAR takes $\left(\mathrm{PAR}_{\min }, \mathrm{PAR}_{\max }\right)$; value range of tone finetuning disturbance of bandwidth bw takes $\left(\mathrm{bw}_{\min }, \mathrm{bw}_{\max }\right)$; the largest number of iterations is NI; the number of new solutions when algorithms are updated once is $M$; parameters are needed by the particle swarm algorithm: inertia weight is $w$; acceleration constants are $c_{1}, c_{2}$; the largest number of iterations is MAXNum; and the particle swarm size is HMS, same as the harmony memory size. 


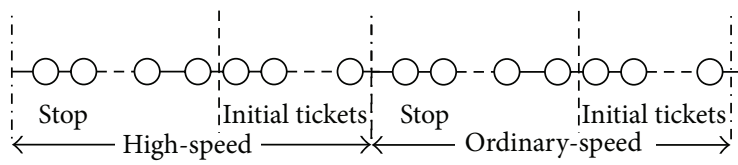

FIGURE 3: Construction of the initial particle.

Step 2 (initial particle is constructed). Initial solution $X$ is the solution vector that consisted of decision variables $x_{i}(i=1,2, \ldots, N)$. Multidimensional ( $W$-dimensional) initial particle is constructed as per the number of variables with each dimension representing the value of each variable of the solution. As is shown in Figure 3, four parts of the particle, respectively, represent stops of different grades trains and initial tickets of sections, with stop using $0-1$ encoding, 0 indicating no stop, and 1 indicating the existence of stop. Tickets are only distributed at stop sections.

Step 3 (constraint conditions are checked). Check constraint conditions as per formulas (10) to (13). If the constraint conditions are not satisfied, update initial particle again.

Step 4 (particle fitness is calculated). Take $F=\left[\sum_{k=1}^{2}\left(M^{k} /\right.\right.$ $\left.\left.\gamma^{k}\right)\right] / R_{\mathrm{OD}}$ as the fitness function.

Step 5 (passenger flow is distributed). Distribute passenger flow as per formulas (1) to (5). Calculate the fitness value based on the new actual passenger flow concluded. Compare the fitness value with that in Step 4 and select the bigger one for the update.

Step 6. Particle formula is updated as

$$
\begin{gathered}
v_{t+1}=w v_{t}+c_{1} * \text { rand }\left(\text { pbest }-x_{t}\right)+c_{2} \\
* \text { rand }\left(\text { gbest }-x_{t}\right), \\
x_{t+1}=x_{t}+v_{t+1} .
\end{gathered}
$$

Repetitively calculate as per Steps 3-6 until the maximum number of iterations and output the optimal value. In the formula, pbest and gbest, respectively, denote the local optimum position and global optimal location of the particle.

The sigmiod function is used to change the trend of inertia weight $w$ evolved with the algorithm. The value interval takes [-9.903438, 9.903438].

Inertia weight decreasing formula is $w=\left[\left(w_{\max }-w_{\min }\right) /\right.$ $\left.1+e^{\left(20 G / G_{\max }-10\right)}\right]+w_{\min }$.

Step 7 (new solution of harmony algorithm is created). The first HMS optimal solutions of particle swarm algorithm from Step 6 are taken as the initial solutions of harmonic algorithm.

(1) Find out the best particle $x$ best and the worst $x$ worst in each generation.

(2) Find out the adaptive bw and PAR according to the current iteration:

$$
\begin{aligned}
\mathrm{PAR} & =\mathrm{PAR}_{\text {min }}+\left(\mathrm{PAR}_{\text {max }}-\mathrm{PAR}_{\min }\right) * \frac{n i}{\mathrm{NI}} \\
\mathrm{bw} & =\mathrm{bw}_{\text {max }} \exp \left(\frac{\log \mathrm{bw}_{\min }}{\mathrm{bw}_{\max }}\right) * \frac{n i}{\mathrm{NI}}
\end{aligned}
$$

(3) bw and PAR are used to find out the current solution of harmony search algorithm. Take the solution as $x_{1 i}$ and judge whether $x_{1 i}$ meets the constraint conditions from (10) to (15); if not, rerun Step 7.

Step 8 (memory library is updated). (1) From 1 dimension to $W$ dimension, randomly select an element of a solution from each dimension. Improve the convergence speed of solution through the reintroduction of particle swarm optimization combined with harmony algorithm. The formula is

$$
x_{t+1}=x_{t}+\operatorname{rand}\left(\text { pbest }-x_{t}\right) \text {. }
$$

Using this formula, find out the current solution, record it as $x_{2 i}$, and judge whether $x_{2 i}$ meets the constraint conditions from (10) to (13); if not, rerun to Step 8.

(2) Compare $f\left(x_{1 i}\right), f\left(x_{2 i}\right)$, and $f$ (xworst), when $f\left(x_{2 i}\right) \geq$ $f\left(x_{1 i}\right) \& f\left(x_{2 i}\right) \geq f(x$ worst $)$, substitute $x_{2 i}$ for $x$ worst, and when $f\left(x_{1 i}\right) \geq f\left(x_{2 i}\right) \& f\left(x_{1 i}\right) \geq f$ (xworst), substitute $x_{1 i}$ for $x$ worst.

Step 9 (iteration is terminated). Determine whether the number of iterations reaches the maximum; if so, stop the iteration and output optimal solutions; otherwise, repeat Steps 7-9.

\section{Analysis of the Example}

The intercity passenger rail corridor as shown in Figure 4 is constructed to verify the above models and algorithms. The relevant parameter calibration is as follows:

(1) throughput capacity: 50 trains/round-the-clock,

(2) travel time value: $E^{T_{1}}=30$ yuan $/ \mathrm{h}, E^{T_{2}}=18.5$ yuan $/ \mathrm{h}$,

(3) transfer time: $t_{m}=15 \mathrm{~min}$,

(4) ticket fare $=$ distance $*$ fare rate,

(5) basic parameters of train operation which are shown in Table 1, 


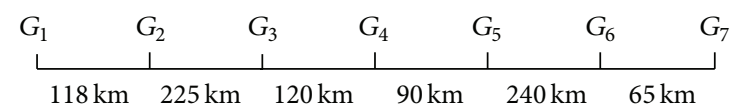

FIGURE 4: Stops and mileage chart of railway passenger transport corridor.

(6) the matrix of the initial passenger flow:

$$
d^{T_{1}}=\left|\begin{array}{ccccccc}
0 & 282 & 1430 & 220 & 765 & 216 & 1638 \\
0 & 0 & 165 & 180 & 200 & 205 & 225 \\
0 & 0 & 0 & 121 & 298 & 168 & 1003 \\
0 & 0 & 0 & 0 & 200 & 185 & 255 \\
0 & 0 & 0 & 0 & 0 & 113 & 1370 \\
0 & 0 & 0 & 0 & 0 & 0 & 202 \\
0 & 0 & 0 & 0 & 0 & 0 & 0
\end{array}\right|,
$$

$$
d^{T_{2}}=\left|\begin{array}{ccccccc}
0 & 560 & 507 & 415 & 580 & 210 & 912 \\
0 & 0 & 465 & 310 & 302 & 251 & 335 \\
0 & 0 & 0 & 281 & 303 & 145 & 522 \\
0 & 0 & 0 & 0 & 395 & 315 & 285 \\
0 & 0 & 0 & 0 & 0 & 448 & 580 \\
0 & 0 & 0 & 0 & 0 & 0 & 457 \\
0 & 0 & 0 & 0 & 0 & 0 & 0
\end{array}\right| .
$$

On the basis of above particle swarm harmony search algorithm, matlab is used for the solution. The optimal solution is found after 400 times of iteration. The matrix of ticket allotment at each section of the station is

$$
\begin{aligned}
\theta^{T_{1}} & =\left|\begin{array}{ccccccc}
0 & 0 & 1811 & 0 & 1281 & 0 & 2398 \\
0 & 0 & 0 & 0 & 0 & 0 & 0 \\
0 & 0 & 0 & 0 & 581 & 0 & 1227 \\
0 & 0 & 0 & 0 & 0 & 0 & 0 \\
0 & 0 & 0 & 0 & 0 & 0 & 1770 \\
0 & 0 & 0 & 0 & 0 & 0 & 0 \\
0 & 0 & 0 & 0 & 0 & 0 & 0
\end{array}\right|, \\
\theta^{T_{2}} & =\left|\begin{array}{lllllllc}
0 & 842 & 440 & 321 & 284 & 206 & 152 \\
0 & 0 & 630 & 490 & 502 & 456 & 560 \\
0 & 0 & 0 & 716 & 258 & 175 & 198 \\
0 & 0 & 0 & 0 & 595 & 500 & 540 \\
0 & 0 & 0 & 0 & 0 & 699 & 280 \\
0 & 0 & 0 & 0 & 0 & 0 & 659 \\
0 & 0 & 0 & 0 & 0 & 0 & 0
\end{array}\right| .
\end{aligned}
$$

TABLE 1: Basic parameters of train operation.

\begin{tabular}{lcc}
\hline Type & High-speed & Ordinary-speed \\
\hline Average travel speed $(\mathrm{km} / \mathrm{h})$ & 250 & 120 \\
Train seating capacity (people) & 610 & 1114 \\
Fare rate (yuan/km) & 0.49 & 0.24 \\
Station dwell time (min) & 2 & 4 \\
\hline
\end{tabular}

Now, 9 high-speed trains are operated with the stop set as $A_{1}=\{1,3,5,7\} ; 4$ ordinary-speed trains are operated with the stop set as $A_{2}=\{1,2,3,4,5,6,7\}$. The total revenue from the fares of intercity trains is 3,049,542 yuan. Passenger flow deviation $R_{\mathrm{OD}}$ is 8322 . When two-grade passengers flow distribution on their own grade trains, respectively, 10 highspeed trains and 5 ordinary-speed trains will be operated to meet the demand of passenger flow. Then the total revenue from the fares of intercity train is 2,997,906 yuan. Through the collaborative optimization of stops and ticket allotment, the total revenue is increased by 51,636 yuan and the numbers of operated high-speed trains and ordinary-speed trains are reduced to 1 , respectively. The average utilization rates of train section reach $98.43 \%$ and $83.09 \%$ which are increased by $14.16 \%$ and $12.52 \%$, respectively.

The example demonstrates that collaborative optimization of the stop schedule plan and ticket allotment of intercity trains of two grades can improve the sectional utilization rate of the train; thus, the vehicle operation costs can be reduced, and the revenue of inter-city railway system can be improved. The effectiveness of the proposed model and algorithm is verified; meanwhile, although the passenger flow conversion caused by the collaborative optimization scheme may reduce the passenger travelling satisfaction, the matching relationship between the revenue of the railway system and the passenger travelling satisfaction can be adjusted by setting the weight parameters between them.

\section{Conclusion}

Train grade of intercity passenger rail corridor, stop schedule plan, and ticket allotment constitute the three elements of the passenger travelling behavior choice. This paper depicts the influence mechanism of the collaborative optimization of the stop schedule plan and ticket allotment on passenger flow conversion in the intercity passenger rail corridor with trains of different grades, based on which, a passenger flow conversion equation is set up and a multiobjective model considering the revenue of the intercity railway system and the passenger travel satisfaction is built up. The model is solved by the particle swarm harmony search algorithm. This updated algorithm sets up a new comparative rule and improves the occurrence probability of the best solution.

The example verifies the effectiveness of the model and algorithm, and it proves that the collaborative optimization of the stop schedule plan and ticket allotment of trains of different grades can improve the sectional utilization rate of the train, increase the revenue of intercity railway system, and realize the optimum matching between system revenue and passenger travelling satisfaction. 


\section{Conflict of Interests}

The authors declare that there is no conflict of interests regarding the publication of this paper.

\section{Acknowledgments}

This work is partly supported by the National Social Science Fund (14XGL011) and Natural Science Fund (1506RJZA062) of Gansu Province, China.

\section{References}

[1] S. E. Kimes, "Yield management: a tool for capacity-constrained service firms," Journal of Operations Management, vol. 8, no. 4, pp. 348-363, 1989.

[2] K. Talluri and G. Van Ryzin, "Revenue management under a general discrete choice model of consumer behavior," Management Science, vol. 50, no. 1, pp. 15-33, 2004.

[3] A. Armstrong and J. Meissner, "Railway revenue management: overview and models," Working Paper, Lancaster University Management School, 2010.

[4] S. Kunnumkal and H. Topaloglu, "A new dynamic programming decomposition method for the network revenue management problem with customer choice behavior," Production and Operations Management, vol. 19, no. 5, pp. 575-590, 2010.

[5] A. Ciancimino, G. Inzerillo, S. Lucidi, and L. Palagi, "A mathematical programming approach for the solution of the railway yield management problem," Transportation Science, vol. 33, no. 2, pp. 168-181, 1999.

[6] P.-S. You, "An efficient computational approach for railway booking problems," European Journal of Operational Research, vol. 185, no. 2, pp. 811-824, 2008.

[7] Y. Bao, The Theory and Methods for Railway Seat Inventory Control, Beijing Jiaotong University, Beijing, China, 2013.

[8] J. Huang and Q. Peng, "Two-stage optimization algorithm for stop schedule plan of high-speed train," Journal of Southwest Jiaotong University, vol. 47, no. 3, pp. 484-489, 2012.

[9] H.-Q. Peng and Y.-J. Zhu, "Intercity train operation schemes based on passenger flow dynamic assignment," Journal of Transportation Systems Engineering and Information Technology, vol. 13, no. 1, pp. 111-117, 2013.

[10] B. Lan and L. Zhang, "Revenue management model for highspeed passenger railway," Chinese Journal of Management Science, vol. 17, no. 4, pp. 53-59, 2009.

[11] X. Shan, Study on Railway Revenue Management Model and Its Application in Passenger Transportation, China Academy of Railway Sciences, Beijing, China, 2012.

[12] F. Shan, Y. Chen, and W. Zhou, "Railway passenger train seats allotment plan establishment and evaluation method based on user equilibrium analysis," China Railway Science, vol. 29, no. 6, pp. 98-103, 2008. 


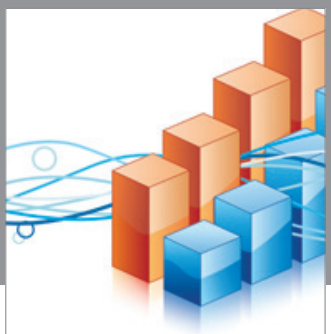

Advances in

Operations Research

vatem alat4

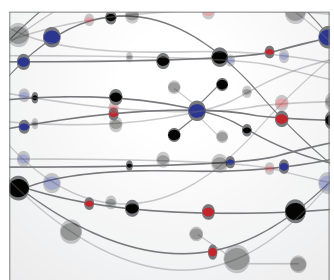

\section{The Scientific} World Journal
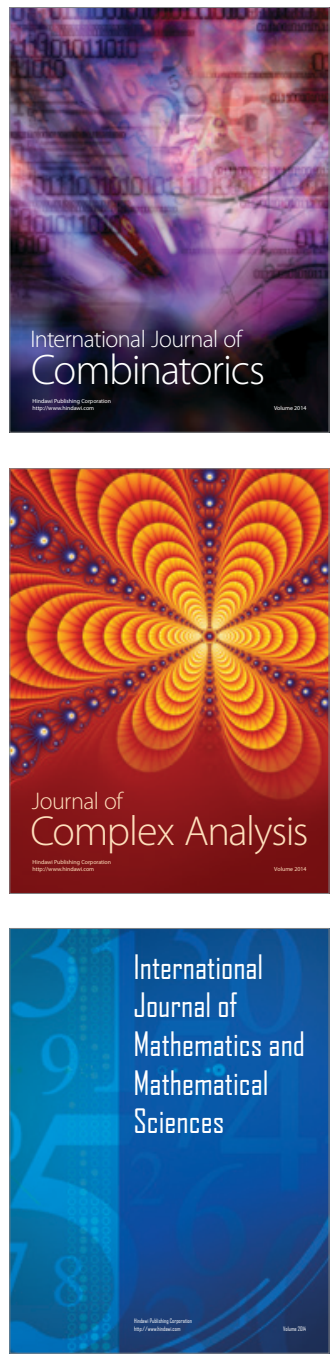
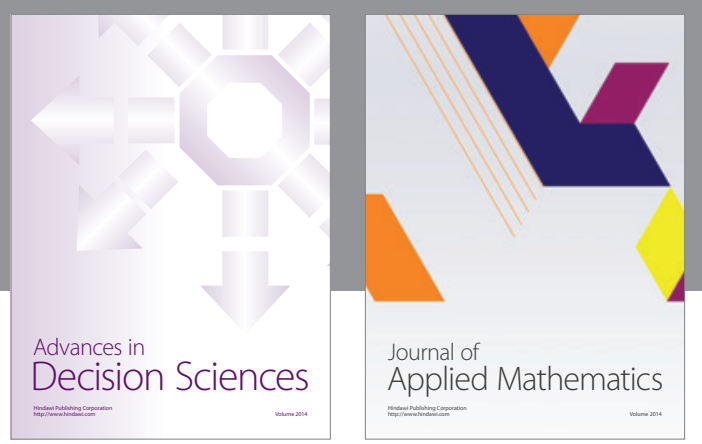

Algebra

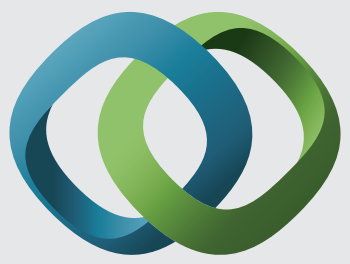

\section{Hindawi}

Submit your manuscripts at

http://www.hindawi.com
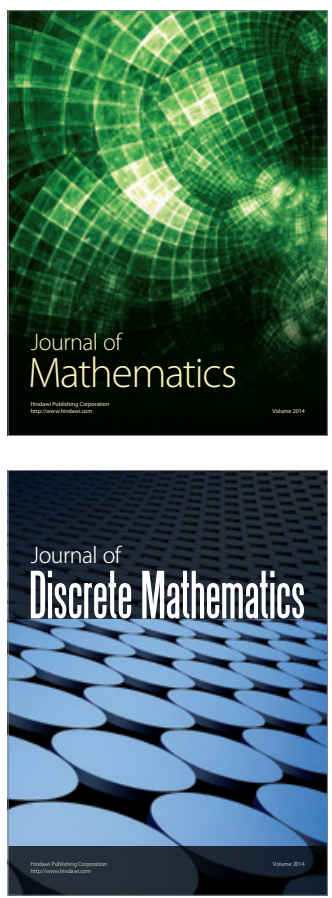

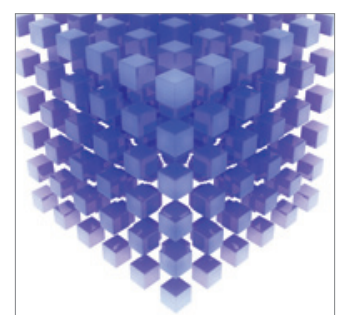

Mathematical Problems in Engineering
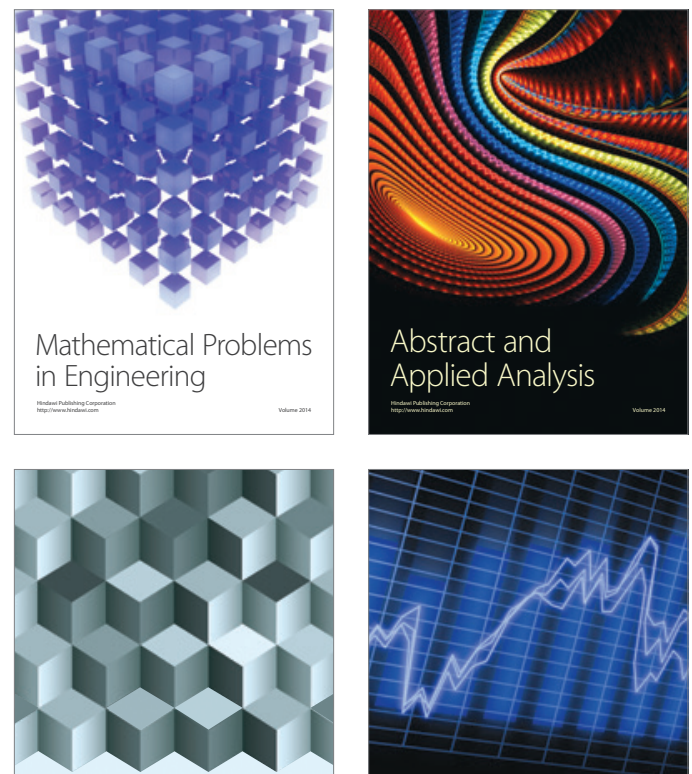

Journal of

Function Spaces

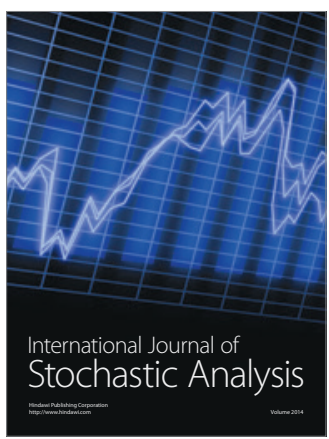

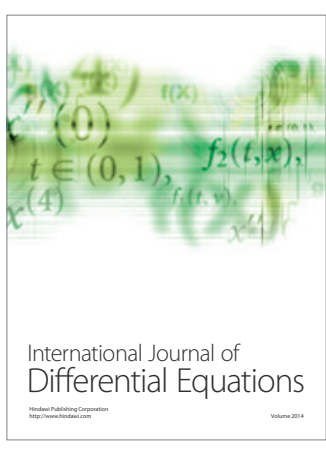
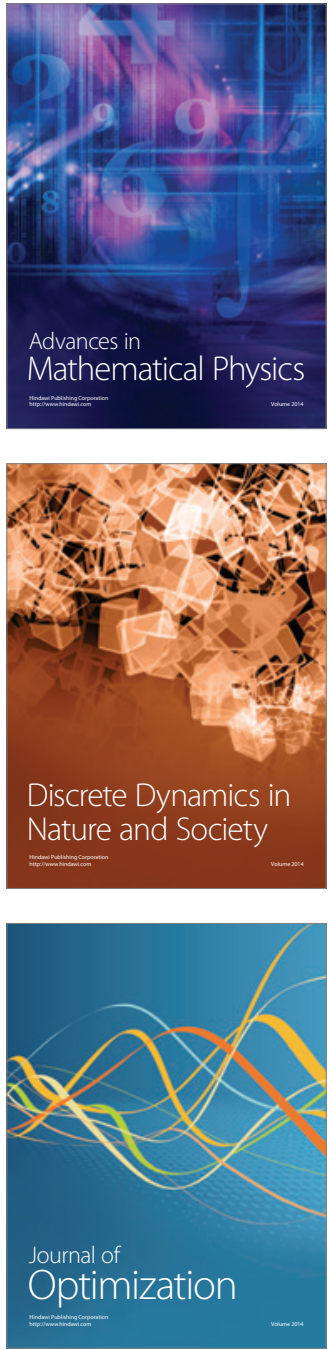